\title{
Fully resolved simulation and ultrasound flow studies in stented carotid aneurysm model
}

J. Mikhal, A. M. Hoving, G. M. Ong, C. H. Slump

J. Mikhal, A. M. Hoving, G. M. Ong, C. H. Slump, "Fully resolved simulation and ultrasound flow studies in stented carotid aneurysm model

," Proc. SPIE 10951, Medical Imaging 2019: Image-Guided Procedures, Robotic Interventions, and Modeling, 1095115 (8 March 2019); doi:

$10.1117 / 12.2513002$

SPIE. Event: SPIE Medical Imaging, 2019, San Diego, California, United States 


\title{
Fully resolved simulation and ultrasound flow studies in stented carotid aneurysm model
}

\author{
J. Mikhal*, A.M. Hoving, G.M. Ong, C.H. Slump \\ TechMed Centre, University of Twente, 7500 AE, Enschede, The Netherlands
}

\begin{abstract}
Introduction. Treatment choice for extracranial carotid artery widening, also called aneurysm, is difficult. Blood flow simulation and experimental visualization can be supportive in clinical decision making and patient-specific treatment prediction. This study aims to simulate and validate the effect of flow-diverting stent placement on blood flow characteristics using numerical and in vitro simulation techniques in simplified carotid artery and aneurysm models.

Methods. We have developed a workflow from geometry design to flow simulations and in vitro measurements in a carotid aneurysm model. To show feasibility of the numerical simulation part of the workflow that uses an immersed boundary method, we study a model geometry of an extracranial carotid artery aneurysm and put a flow-diverting stent in the aneurysm. We use ultrasound particle image velocimetry (PIV) to visualize experimentally the flow inside the aneurysm model.

Results. Feasibility of ultrasound visualization of the flow, virtual flow-diverting stent placement and numerical flow simulation are presented. Flow is resolved to scales much smaller than the cross section of individual wires of the flowdiverting stent. Numerical analysis in stented model introduced $25 \%$ reduction of the blood flow inside the aneurysm sac. Quantitative comparison of experimental and numerical results showed agreement in 1D velocity profiles.

Discussion/conclusion. We find good numerical convergence of the simulations at appropriate spatial resolutions using the immersed boundary method. This allows us to quantify the changes in the flow in model geometries after deploying a flow-diverting stent. We visualized the physiological blood flow in a 1-to-1 aneurysm model, using PIV, showing a good correspondence to the numerical simulations. The novel workflow enables numerical as well as experimental flow simulations in patient-specific cases before and after flow-diverting stent placement. This may contribute to endovascular treatment prediction.
\end{abstract}

Keywords: aneurysm, carotid artery, flow-diverting stent, numerical simulations, ultrasound PIV, 3d printed geometry

*j.mikhal@utwente.nl; phone 0031534898017

\section{INTRODUCTION}

Extracranial carotid artery widening, also called aneurysm (ECAA), is a rare disease ${ }^{1,2}$. Risk of rupture, TIA, stroke or large swelling and therefore patient discomfort, indicate in some cases the need for treatment. ECAA can be treated conservatively, by applying best medical treatment, with open surgery or minimally invasive surgery, or by a combination of two or more of these treatment types ${ }^{3}$. Due to the rarity of this disease, the optimal treatment is unknown ${ }^{3}$. In intracranial arteries, but also in peripheral arteries, a flow-diverting stent is clinically applied to exclude the aneurysm sac from blood flow and approximately restore the original blood flow ${ }^{4,5}$. Only a few patient cases describe flow-diverting stent placement in $\mathrm{ECAA}^{6,7}$.

The effect of stent placement can be estimated before intervention by in vitro or in silico experiments. Several visualization techniques are available to perform in vitro flow experiments. These techniques use medical imaging techniques, such as CT angiography, MR angiography or Doppler ultrasound, or optical techniques, such as laser particle image velocimetry (PIV). A novel medical-based flow study technique is ultrasound PIV. In vitro studies ${ }^{8,9}$, as well as first clinical studies ${ }^{10,11}$, show the wide applicability of this technique. Also, in vitro validation of ultrasound PIV in the presence of a stent was recently performed ${ }^{12}$. Therefore, ultrasound PIV is a promising technique for in vitro investigation of flow patterns in ECAA before and after stent placement.

Medical Imaging 2019: Image-Guided Procedures, Robotic Interventions, and Modeling, edited by Baowei Fei, Cristian A. Linte, Proc. of SPIE Vol. 10951, 1095115 · C 2019 SPIE CCC code: $1605-7422 / 19 / \$ 18 \cdot$ doi: $10.1117 / 12.2513002$ 
Besides in vitro studies, computational hemodynamics modeling can also be helpful in predicting intervention outcome ${ }^{13-}$ ${ }^{15}$. Numerical blood flow simulations are performed widely in all types of human arteries ${ }^{16-18}$. The precise geometry of the pathological vessel and the actual condition of the patient are required for fluid mechanical analysis. There are several methods to represent the geometry as well as various computational fluid dynamics software available for simulations of blood flow. However, simulation after stent placement is time consuming, due to the fine structures of the stent, prompting the need for very high spatial resolution. Moreover, correct simulation of stent fitting in the arterial model is challenging ${ }^{18,19}$. We use the immersed boundary method for precise representation of the 3D geometry with and without flow-diverting stent placed. Fluid mechanical analysis is implemented using the open source software OpenFOAM.

The final goal of this project is to develop and validate a workflow to perform flow analysis in patient-specific ECAA to evaluate endovascular treatment options and outcomes. In this paper we focus on the development of the workflow in one simplified ECAA model. The workflow comprises the use of 3D modelling and printing techniques for model design for two distinctive flow analysis techniques. Results of flow analysis in both numerical simulations and ultrasound experiments are presented in this paper. We also focus on virtual flow-diverting stent placement and flow resolved numerically in a model containing this stent.

\section{METHODS}

We report on a workflow for analyzing flow dynamics associated with untreated and stented aneurysms. We performed a literature search to find ECAA types and morphologies. Based on the most common morphology, we designed a 3D aneurysm model using Meshmixer 3.4.35 (Autodesk, Inc.). Further, we focus on the process of phantom fabrication and the setup of an ultrasound experiment for visualization of the blood flow inside the model aneurysm. Subsequently, we introduce the immersed boundary geometry and settings for the numerical simulations, as well as virtual placing of the flow-diverting stent inside the carotid aneurysm.

\subsection{Workflow design}

The workflow is shown in Figure 1 and consists of several steps. A model geometry is designed based on a common carotid aneurysm morphology as reported in literature. The design, post-processing and STL export are performed in Meshmixer. Then, the model is used for two purposes: a. numerical simulations and b. ultrasound experiments. For the numerical simulations, flow-diverting stent placement is performed in the STL model using Blender 2.79 (Blender Foundation, Amsterdam, the Netherlands). The STL models - with and without flow-diverting stent - are transferred to a 3D Cartesian grid using especially designed Matlab scripts (Matlab 2017b, The Mathworks Inc., Natick, MA, USA). In this way a 3D masking function is obtained, which is needed for the immersed boundary method. Further, numerical simulations are performed under realistic patient conditions in OpenFOAM. Later, the flow results are analyzed and visualized, using postprocessing steps. For the ultrasound visualization experiment, a 3D print of the aneurysm model is used as mold for casting the poly(vinyl alcohol) (PVA)-phantom. A flow-diverting stent can be placed in the 3D printed model. The in vitro flow experiments are performed using high-frame-rate contrast-enhanced ultrasound techniques ${ }^{12}$.

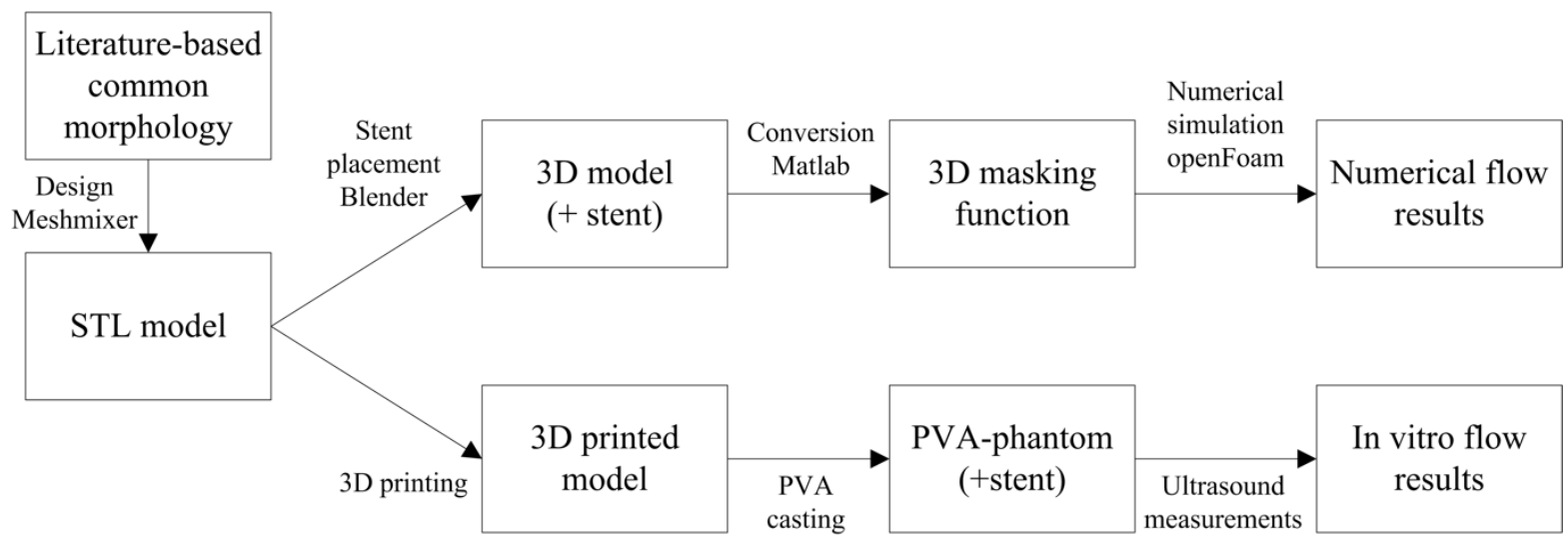

Figure 1. Flow chart of the workflow from geometry design to flow simulations and in vitro results. 


\subsection{Model geometry}

ECAA can be stratified into five types according to the morphology of the aneurysm ${ }^{20}$. A patient study in 48 cases showed 'aneurysm isolated to the internal carotid artery' (type I/II according to the Attigah classification) to be the most common morphology $(65 \%)^{2}$. Based on these findings, a 3D model that captures the main features of a type I aneurysm (Figure 2A) is designed. The 3D STL-model for the carotid aneurysm (Figure 2B) is the basis for the above-described workflow. A 3D-print of the model in 1-to-1 physical dimensions is used for further flow visualization (Figure 2C). Also, the model is translated into the masking function used for numerical simulations of the blood flow (Figure 2D).

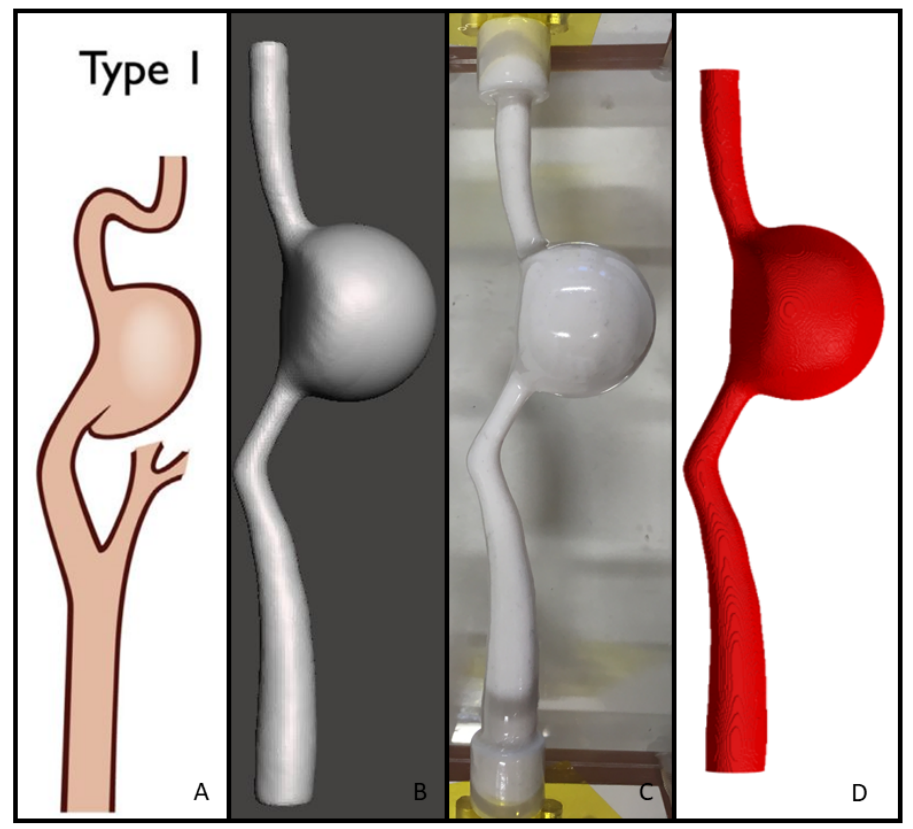

Figure 2. A. Drawing of type I extracranial carotid artery aneurysm, adopted from Nordanstig et al. ${ }^{2} \mathbf{B}$. Model geometry based on type I geometry in 3D STL format. C. 3D-printed carotid aneurysm model. D. 3D masking function geometry.

\subsection{Phantom fabrication}

The model geometry is 3D-printed from P430-ABSPlus White (340-21201, Stratasys) and SR-30 Soluble support (34030500 , Stratasys) on the outside of the model, using a Stratasys Fortus $250 \mathrm{mc}$ printing system. Dimensions of the model are: length $106 \mathrm{~mm}$; diameter inlet $8 \mathrm{~mm}$; diameter outlet $5 \mathrm{~mm}$; aneurysm sac $25 \times 25 \times 21 \mathrm{~mm}^{3}$. To provide connection of the phantom to the flow circuit, tube connections are glued to both the inlet and outlet side of the model (red boxes Figure 3A). The mold - the 3D-printed model plus tube connections, placed in a plexiglass box (Figure 3B) - is filled with a PVA-mixture (Figure 3C). This mixture contains 10\%wt PVA-powder (mw 85,000 - 124,000, 99+\% hydrolyzed, Aldrich Chemistry) dissolved in cooling liquid (G12+ longlife Coolant, All Ride), which is stirred at $90^{\circ} \mathrm{C}$ for 3 hours. Before pouring, the mixture is placed in vacuum for one hour to remove air bubbles. The phantom underwent three freeze-thaw cycles (17 hours freezing; 9 hours thawing). The 3D-printed model is removed from the phantom by 5 hours of acetone flushing (Figure 3D). 


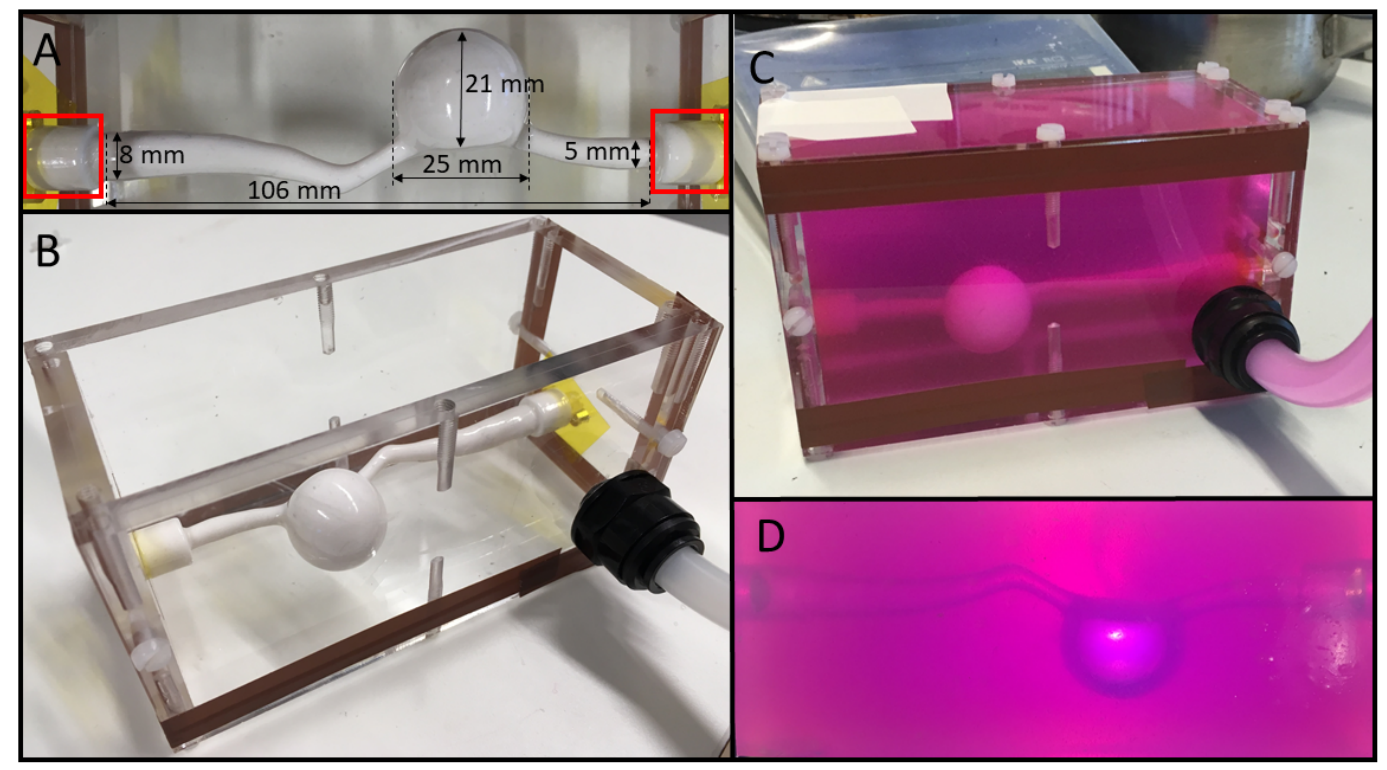

Figure 3. A. 3D-printed model geometry. Red boxes: tube connections. B. 3D-printed model geometry in plexiglass mold. C. 3D-printed model geometry in mold after pouring the PVA-mixture. D. PVA-phantom after removing the 3D-printed model.

\subsection{Ultrasound experiment}

An ultrasound experiment is performed to visualize and quantify flow patterns in the carotid artery aneurysm phantom using the setup and post-processing as described in Hoving et al. ${ }^{12}$. The setup and settings are slightly adjusted for this experiment (Figure 4). Blood mimicking fluid (glycerol - water mixture $\left.(39 \% \mathrm{wt}-61 \% \mathrm{wt}), v=3.3 \times 10^{6} \mathrm{~m}^{2} / \mathrm{s}\right)$ is pumped through the phantom with a continuous flow rate of $0.390( \pm 0.05) \mathrm{L} / \mathrm{min}$, using an electric gear pump $(1.8 \mathrm{~L} / \mathrm{min} 12 \mathrm{~V}$, KAVAN GmbH, Nürnberg, Germany) and an ultrasonic flow meter (3\% accuracy, UF Ultrasonics Flow Meter, Cynergy3 Components, UK). This flow rate corresponds to a mean velocity of $0.23( \pm 0.018) \mathrm{m} / \mathrm{s}$ in a $6 \mathrm{~mm}$ diameter vessel (approximately mean diameter of phantom without aneurysm). Laminar flow is assumed, since the Reynolds number is approximately 400. Images are obtained with 5022 frames per second using angled plane wave acquisition on a Verasonics Vantage 256 system (Verasonics Inc., Kirkland, WA, USA). The effective frame rate is 837, since one frame is the result of two subapertures (due to only 96 receive channels in L12-3v transducer) and three angled plane wave transmits. The length of the phantom is larger than the width of the L12-3v transducer, so acquisition in three positions is necessary to capture the total phantom. PIV-analysis is applied on the three imaging sets, using interrogation windows of $3.2 \times 1.5 \mathrm{~mm}^{2}$ $(16 \times 16$ pixels $)$ followed by $1.6 \times 0.75 \mathrm{~mm}^{2}(8 \times 8$ pixels $)$ in an iterative manner. The velocity information is averaged over the first 100 frames, corresponding to a period of 0.12 seconds. Five positions in the phantom are selected to compare the results of the ultrasound and simulation experiments.

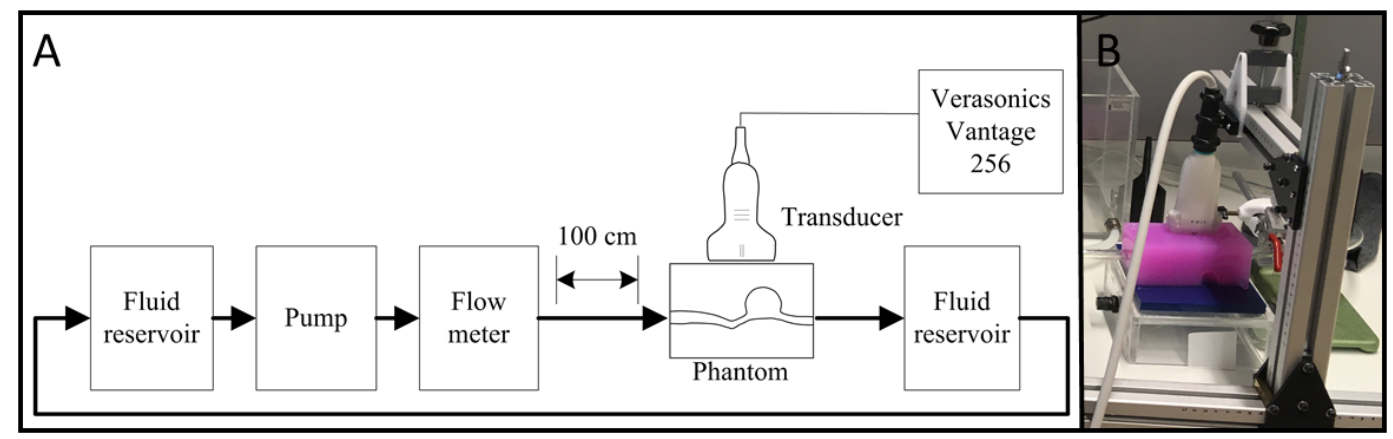

Figure 4. A. Schematic view of ultrasound experiment setup. B. Photograph of PVA-phantom and L12-3v transducer in ultrasound experiment setup. 


\subsection{Computational model}

The numerical model for the simulation of the blood flow in the carotid artery is based on the incompressible NavierStokes equations in 3D. To capture the flow in the aneurysm geometry an immersed boundary method is used, which is based on a volume penalization approach ${ }^{21}$. Thus, complex geometries can be represented on a 3D Cartesian grid using a so-called masking function. This masking function is equal to 0 inside the fluid part of the computational domain and equal to 1 inside the solid parts of the domain. Direct transformation from STL format into the masking function delivers a relatively fast tool to represent this rather complex geometry, even with the flow-diverting stent placed inside. This approach does not require additional steps, such as smoothing or local grid refinement. The resulting masking function for the carotid aneurysm model is shown in Figure 5.

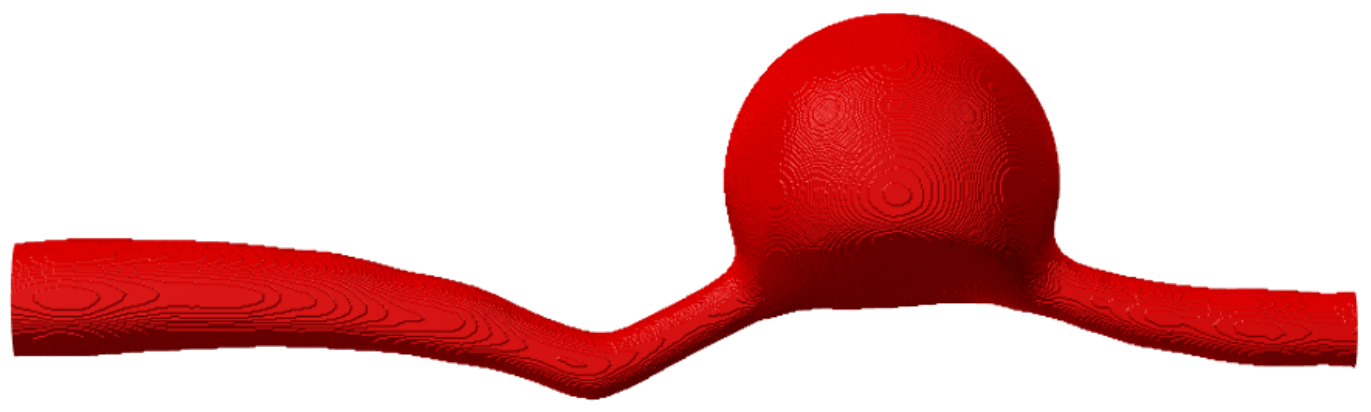

Figure 5. 3D immersed boundary masking function of the carotid aneurysm model. Grid resolution is $2000 \times 720 \times 600$.

Virtual flow-diverting stent placement is performed in the computational model using Blender software (Figure 6ABC). First, the flow-diverting stent is placed roughly in place, after which the stent is deformed smoothly to accommodate the aneurysm geometry. Subsequently, the model is converted into the immersed boundary method's masking function using Matlab (Figure 6D).

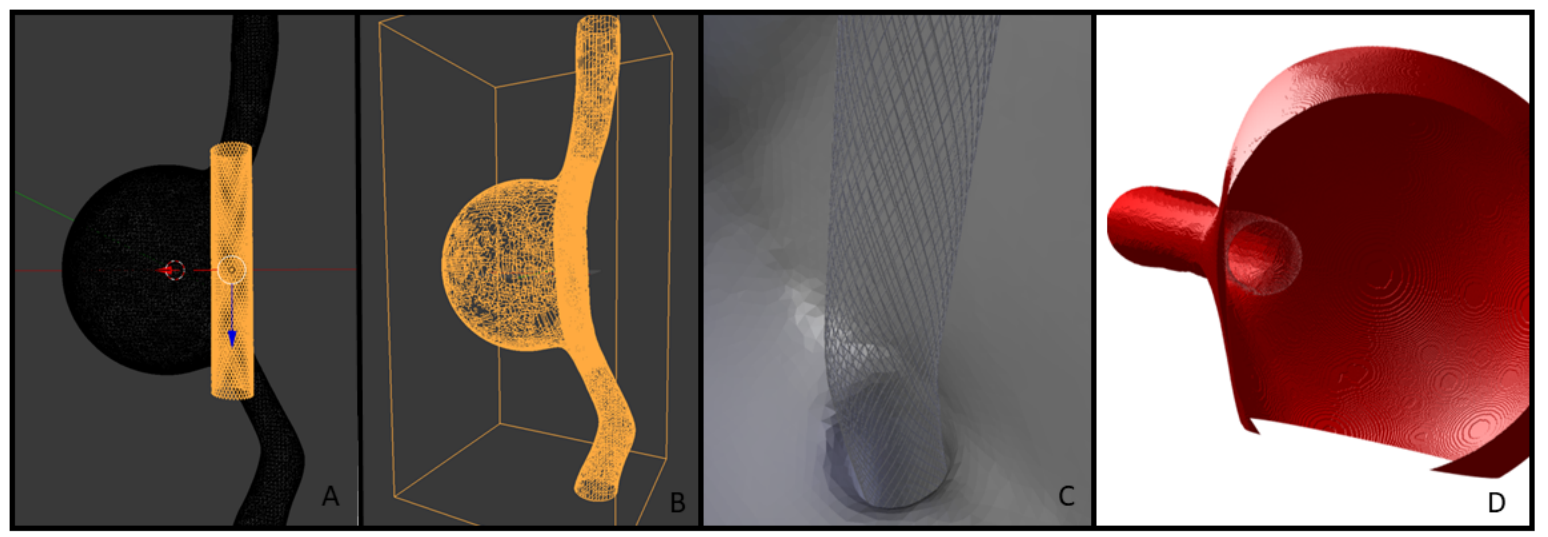

Figure 6. A. Coarse positioning of the flow-diverting stent. B. Flow-diverting stent adapted to the model carotid artery, shown as region of more dense grid. C. Flow-diverting stent inside the aneurysm cavity - view in Blender. D. Masking function of the aneurysm with flow-diverting stent inside.

The physical dimension of the total model geometry are $100 \mathrm{~mm} \times 36 \mathrm{~mm} \times 30 \mathrm{~mm}$, while the thickness of the wires of the flow diverter is $0.03 \mathrm{~mm}$. Representation of all these physical scales - the relatively huge domain with the rather tiny wires of the flow-diverting stent in it - is a challenge for the resolution of numerical simulations, as the flow around every thin wire of the flow-diverting stent has to be properly captured. This implies a correspondingly high spatial grid resolution and computational expense. Thus, for the geometry without flow-diverting stent we use a grid resolution of $400 \times 144 \times 120$, while with stent we use the grid of $800 \times 288 \times 240$. 
Numerical simulations are performed in the open source software OpenFOAM, using the icoFoam solver for incompressible fluids. Similar to the ultrasound experiment, flow at Reynolds number 400 is considered, which is motivated by the physical dimensions and realistic carotid blood flow characteristics. An average diameter of the carotid artery of $6 \mathrm{~mm}$ is considered and the average velocity appeared to be around $0.23 \mathrm{~m} / \mathrm{s}$ for the blood with a viscosity of $3.3 \times 10^{-6} \mathrm{~m}^{2} / \mathrm{s}$. For the results presented in this paper, inflow and outflow conditions are used, in which a steady uniform velocity profile is applied at the inflow.

\section{RESULTS}

We first present results of ultrasound visualization of the blood flow inside the carotid artery aneurysm. Further, the flow obtained from numerical simulations is analyzed and the modification of the flow due to placement of the flow-diverting stent is quantified. Finally, the in vitro and numerical results without a flow-diverting stent are compared for the flow at several locations through the model geometry of ECAA.

\subsection{Ultrasound experiment}

Microbubble signals are clearly visible after singular value decomposition based filtering of the coherently compounded imaging frames. Velocity peak values increase after the bending of the inflow artery until halfway of the aneurysm sac (Figure 7). From there, a straight flow jet continues until it hits the downstream side of the aneurysm, where it splits. One part distributes into the aneurysm sac, and the other part flows into the outlet artery. The disturbed flow patterns inside the aneurysm sac are better visible in the imaging frames over time (Video 1).
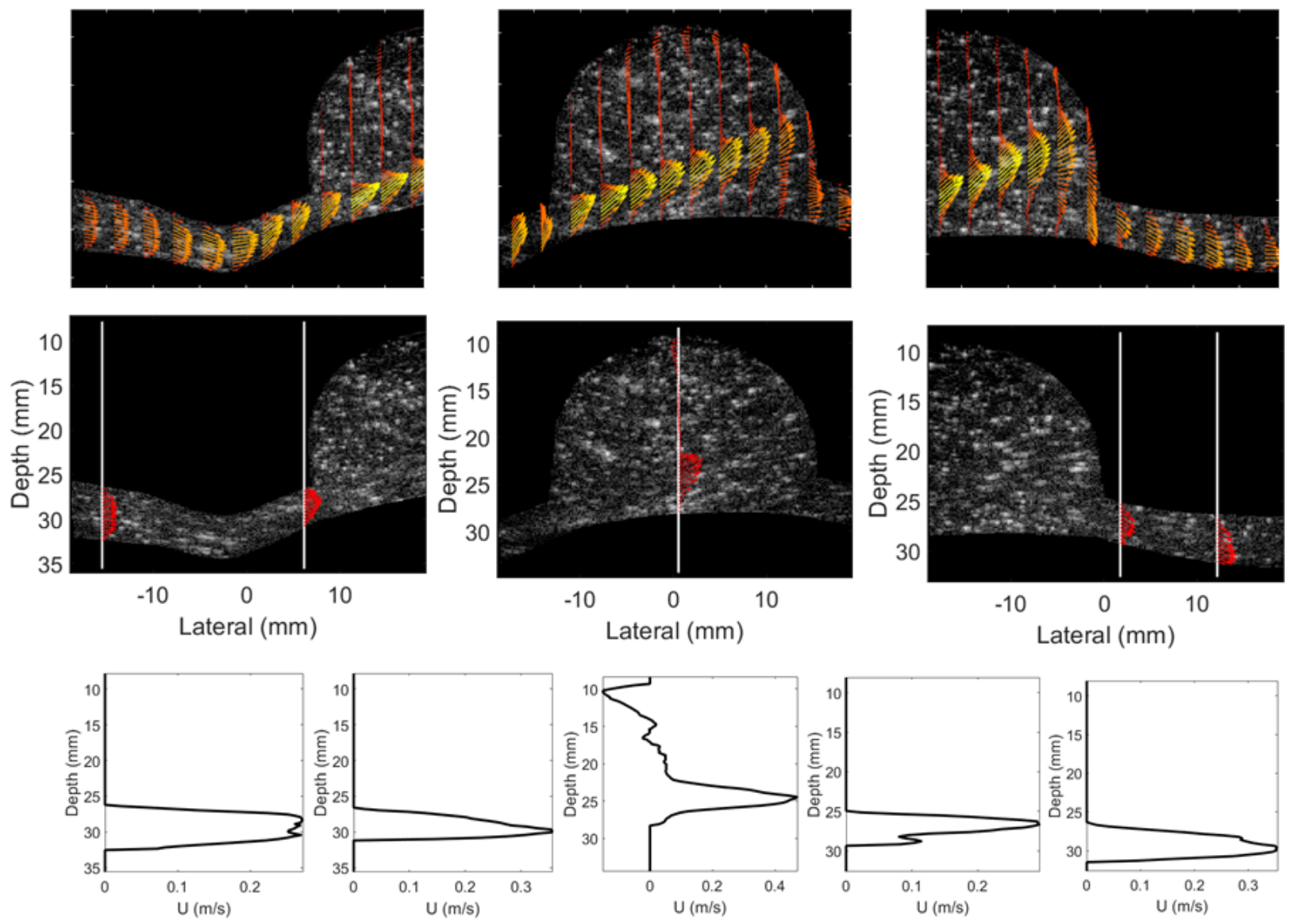

Figure 7. Top row: ultrasound images recorded at three positions with averaged velocity profiles obtained after PIV-analysis. Middle row: velocity profiles on five selected cross-sections. Bottom row: graphical view of the velocity profile at the five selected cross-sections. The velocity profiles are extracted from the signal using PIV analysis software implemented in Matlab. The background of the ultrasound images (pixels outside the region of interest) is colored black. 


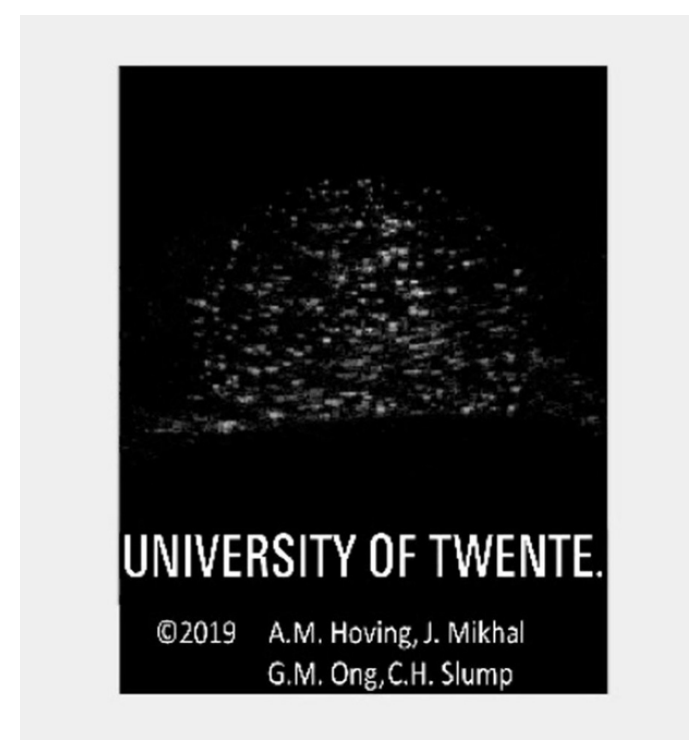

Video 1. Result of filtered ultrasound images showing microbubble signals flowing through the aneurysm sac. A jet flow crosses the aneurysm sac and counterclockwise rotating flow in the aneurysm is visible. The video contains 250 subsequent imaging frames and is recorded with 30 frames per second. http://dx.doi.org/10.1117/12.2513002.1

\subsection{Numerical simulations in (stented) aneurysms}

A cross section through the model geometry showing the magnitude of the velocity field without flow-diverting stent is presented in Figure 8. At this cross-section through the geometry, we observe a jet detaching and hitting the downstream wall of the aneurysm sac, thereby creating a region of high wall shear stresses locally. This flow structuring is clearly similar to the experimental observations in Figure 7. The placement of the flow-diverting stent (Figure 9) clearly modifies the flow. The jet is seen to deflect slightly upward and the passage of the original jet through the mazes of the stent leads to a reduction of the flow velocity by about $25 \%$. The flow-diverting stent reduces the strength of the jet hitting the aneurysm wall, while allowing sufficient transparency for the overall flow in the carotid artery to be maintained.

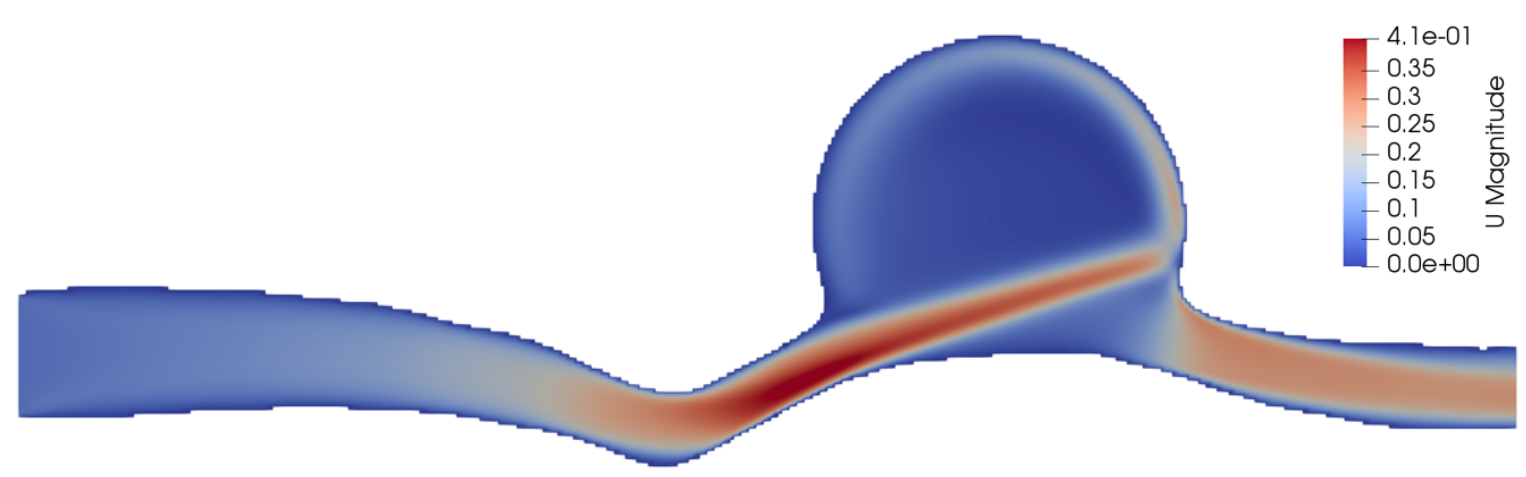

Figure 8. Result of a numerical simulation in the model carotid aneurysm showing high (red) and low (blue) velocity regions, as well as a large vortex in a cross-section of the model. Grid resolution of the entire domain is $400 \times 144 \times 120$, allowing 4 grid points per $1 \mathrm{~mm}$. In red the velocity up to $0.41 \mathrm{~m} / \mathrm{s}$ is represented while dark blue corresponds to stagnant flow with velocity equal to zero. Considerable parts of the aneurysm show a rather slow counterclockwise rotating flow. 


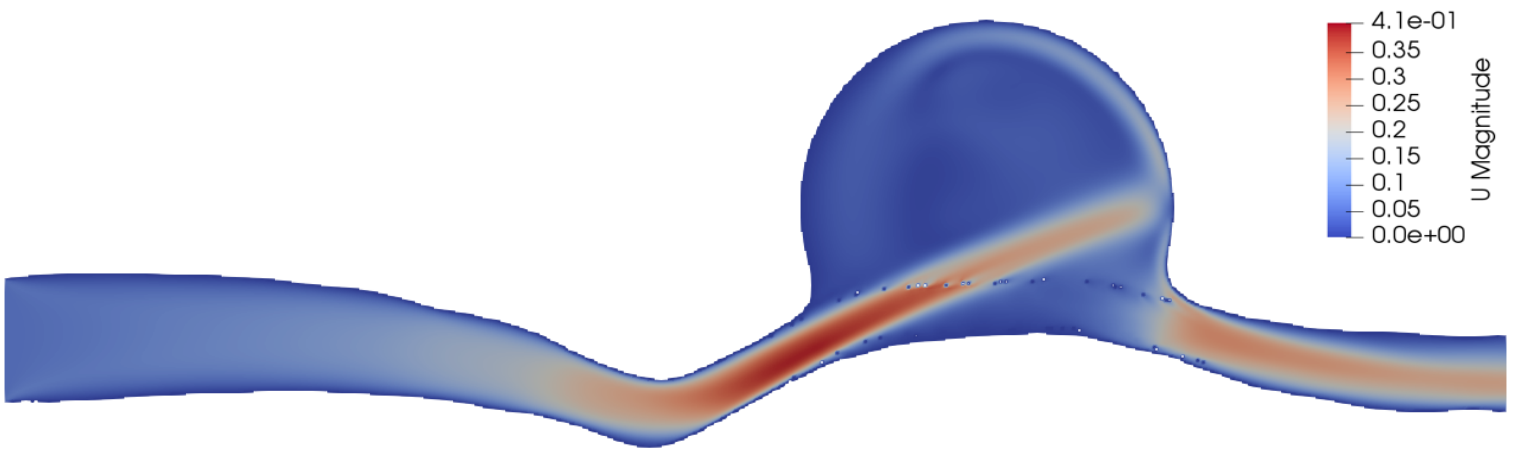

Figure 9. Result of a numerical simulation in the model carotid aneurysm with the flow-diverting stent showing high (red) and low (blue) velocity regions, as well as vortices in a cross-section. Flow is clearly reduced by the presence of the flow-diverting stent, which is in this cross-section visible by the dots. Grid resolution of this simulation is $800 \times 288 \times 240$, allowing 8 grid points per $1 \mathrm{~mm}$. Velocity up to $0.41 \mathrm{~m} / \mathrm{s}$ is represented in red, while dark blue corresponds to stagnant flow with velocity equal to zero. Passage of the jet slows down the flow by about $25 \%$.
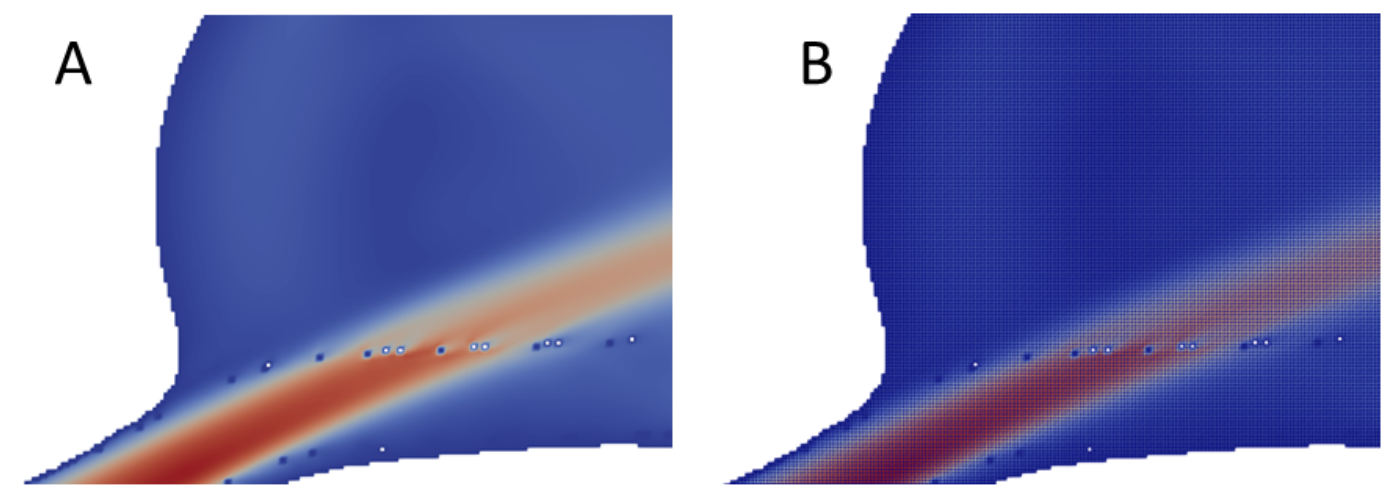

Figure 10. A. Zoomed-in 2D velocity field around the wires of the flow-diverting stent. B. Representation of the flow velocity magnitude including the grid. The resolution of this simulation is $800 \times 288 \times 240$, allowing 8 grid points per $1 \mathrm{~mm}$.

Figure 10 shows a zoomed impression of the model geometry and the simulated flow with the flow-diverting stent in place, highlighting the reduction of the flow inside the aneurysm sac. The effect of the individual wires on the flow is resolved and the modification of the velocity field is clearly visible. The interrupted flow near the wires of the implant is shown in the form of small regions of separated flow.
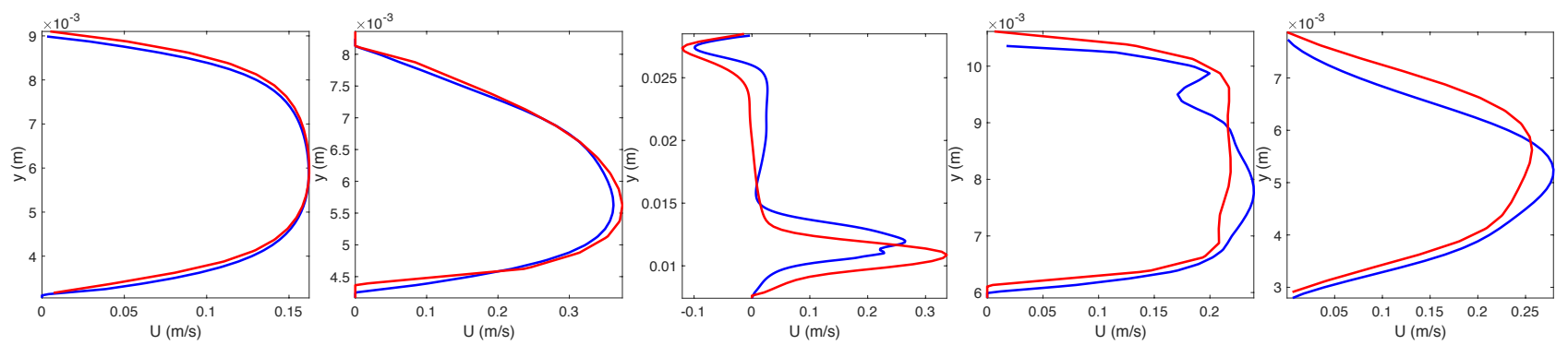

Figure 11. 1D velocity profiles at the 5 selected locations for an aneurysm without stent in red and with virtual stent implanted in blue. Inside the aneurysm sac (middle figure) the reduction of about $25 \%$ of the peak velocity is seen, as the flow passes the stent. Upstream of the aneurysm sac (left two figures) the presence of the aneurysm sac cannot be seen in the flow profiles. Downstream of the aneurysm sac (right two figures) the impact of the stent is seen to reduce with downstream distance as the flow recovers. 
We look further into 1D velocity profiles for the model geometry without and with the flow-diverting stent. In Figure 11 we consider the same 5 locations of interest as considered in the experiment: upstream of the aneurysm, inside, and downstream. Thus, for flow profiles upstream of the aneurysm sac there is almost no difference in the velocity profiles, as expected since the stent is not present at those locations. Inside the aneurysm we observe that the virtually implanted stent reduces the peak flow velocity by about $25 \%$ compared to the case without a stent. Two more profiles are taken in the domain downstream of the aneurysm sac, where we can observe how the flow recovers after going through flow-diverting stent. There are clearly some changes in the flow directly behind the aneurysm sac, while further away we observe quite similar profiles again irrespective of the presence of a stent or not.

\subsection{Comparison of experimental and numerical flow profiles}

To compare experimental and numerical results for the model geometry of ECAA, we first look into 2D velocity profiles to get a qualitative impression (Figure 12). In both cases we observe a jet crossing the aneurysm sac, inducing a counterclockwise rotating flow in the aneurysm sac, in this center cross section. Further the flow proceeds into the downstream vessel.
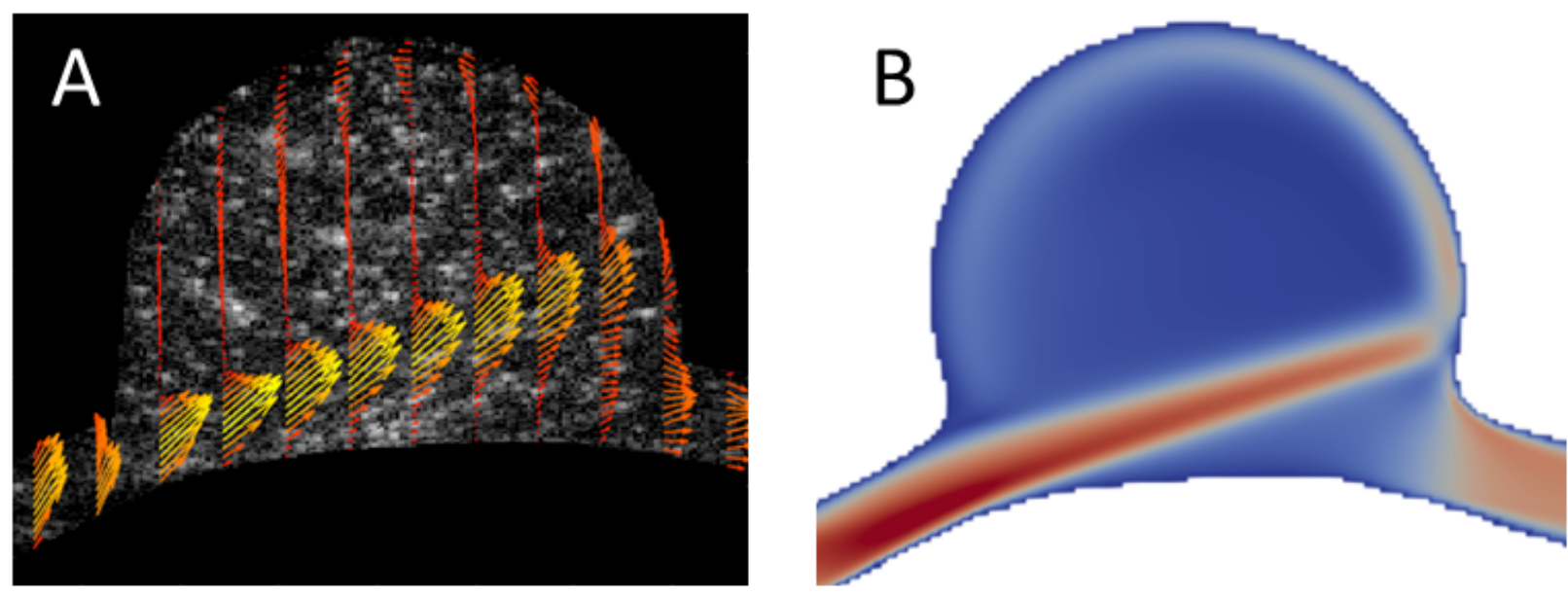

Figure 12. A. PIV analysis of the velocity profile inside the aneurysm. B. Cross-section through the aneurysm showing the numerically obtained magnitude of the flow field.

For quantitative comparison we analyze further 1D velocity profiles. Due to some uncertainty about the precise region of the flow domain sampled by the ultrasound transducer, a direct comparison between experimental and numerical findings is slightly hampered. In fact, the plane in which the device measures the velocity may be tilted vertically, and also the exact downstream location of measurement is known with some uncertainty. To compensate for this, we scaled the observed numerical velocity profile at the left-most location such that the peak value agrees with the experimental observation. In Figure 13A we notice that the width of the flow profiles correspond closely with each other, establishing a good degree of experimental and numerical accuracy. Using the same scaling factor, we see good correspondence of the velocity profile across the entire aneurysm (Figure 13B). Forward and backward flow are clearly present inside and near the upper wall of the aneurysm sac respectively. The uncertainties mentioned regarding the sampled region by the ultrasound transducer are clearly illustrated by the difference in computational domain size by the experiment relative to the precise numerical value. Nevertheless, there is an excellent agreement between experiment and simulation regarding the widths of the forward and backward flow structures and their velocity magnitudes. 

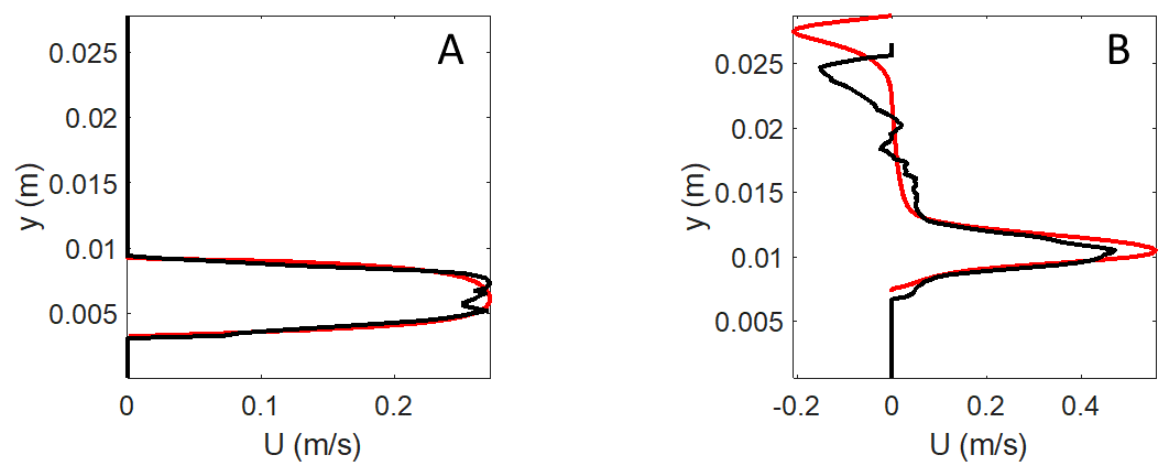

Figure 13. A. 1D velocity profile at location 1 - upstream of the aneurysm. Numerical profile (red) is scaled to the experimental (black). B. 1D velocity profiles in the middle of the aneurysm at location 3. Peak of the jet inside the aneurysm as well as the vortex and back flow are present in both experimental (black) and numerical (red) profiles. There is a difference of about $10 \%$ in the perceived upper wall of the aneurysm sac due to measurement uncertainties.

\section{DISCUSSION AND CONCLUSIONS}

A comprehensive workflow for blood flow simulations in a 3D carotid artery aneurysm model is presented. The workflow comprises 3D model design, and an experimental part with phantom fabrication and in vitro ultrasound flow experiments, next to a numerical part of blood flow simulations, with and without virtual flow-diverting stent placement. Endovascular treatment options and outcomes directly related to hemodynamics can be analyzed using this workflow. Moreover, this workflow enables comparison before and after stent placement in any 3D geometry and gives the possibility to validate numerical and experimental simulations.

Results of the ultrasound experiment and numerical flow simulations in the same model show qualitatively and quantitatively similar flow patterns. A jet flow goes into and across the aneurysm sac and strikes the downstream wall, from where it splits into a stream into the aneurysm sac and one toward the outflow artery. A counterclockwise vortex is visible in the aneurysm, produced by the fluid that is deflected into the aneurysm sac. Quantitative comparison of the velocity values results in similar peak velocities in both the numerical and ultrasound experiment. This indicates that detailed comparison between the two types of flow study is possible. Moreover, virtual stent was placed in the aneurysm STL-model and realistic flow simulation results were obtained at resolutions that capture details down to the scale of the diameter of individual stent wires, using the immersed boundary method. As expected, the numerical simulations show a reduction of the flow velocity into the aneurysm after flow-diverting stent placement by about $25 \%$.

There are some challenges and limitations regarding the correspondence between the numerical and experimental findings. First, because no actual stent was available, only the clean case without stent could be used for comparison. Moreover, even if a commercial stent would be placed, there could remain some uncertainty as to the exact location the stent was deployed, compared to the numerical model. Second, we observe some differences in geometry of the ultrasound phantom and the original 3D model. For example, the maximum diameter of the aneurysm sac in the ultrasound images is $19 \mathrm{~mm}$, while this is $21 \mathrm{~mm}$ in the actual model and in the masking function of the numerical study. This difference might be due to slow and limited shrinking of the phantom after the freeze-thaw procedure. Similarly, some uncertainty arises regarding the precise positioning of the ultrasound transducer. This could also explain the discrepancies reported in Figure 13B, where we compare numerical and experimental results. Third, ultrasound acquisition is performed in 3 subsequent positions, because the transducer did not have enough length to capture the whole phantom. Fourth, an important challenge in the workflow is the high spatial resolution of the masking function required for accurate prediction of the flow around the wires of the flow-diverting stent. We used a grid resolution of $400 \times 144 \times 120$ for the geometry without a stent and a resolution of $800 \times 288 \times 240$ with the flow-diverting stent. This implies $4-8$ grid points per $1 \mathrm{~mm}$, i.e., covering the diameter of the carotid artery with over $25-50$ grid cells, which is proven to capture the structures of the flow ${ }^{19,21}$. To catch the geometry with the flow-diverting stent, we need to considerably increase the amount of grid points in order to resolve the structure of the flow-diverting stent as well. The resolution in the range of $2000 \times 720 \times 600$ or higher, i.e., 20 grid points 
or more per $1 \mathrm{~mm}$, would imply about one grid cell or more across the thickness of a wire in the stent. This would make simulations quite costly. To give an indication of the costs: we typically need 150 CPU-days to reach a steady solution.

Future work will focus on the further validation of the complete workflow using more geometries of patient-specific cases. Also, the comparison between ultrasound experiments and numerical simulation will be extended including more locations (experiment) and more spatial resolutions (simulation). Additionally, flow-diverting stent placement will be conducted in the phantom as well. Ideally, to validate the workflow for intervention prediction, experiments need to be performed using several stent types and more (patient-specific) geometries. The use of 3D modelling and printing in our workflow enables conversion of patient data (CT angiography, MR angiography) to masking functions and ultrasound phantoms.

\section{ACKNOWLEDGMENTS}

The authors would like to thank Henny Kuipers for his contributions to the fabrication of phantom and the flow setup.

\section{REFERENCES}

[1] El-Sabrout R. and Cooley D.A. "Extracranial carotid artery aneurysms: Texas Heart Institute experience," J Vasc Surg. 31(4), 702-712 (2000).

[2] Nordanstig J., Gelin J., Jensen N., Österberg K., Strömberg S., "National experience with extracranial carotid artery aneurysms: Epidemiology, surgical treatment strategy, and treatment outcome," Ann Vasc Surg. 28(4), 882-886 (2014).

[3] Welleweerd J.C., Den Ruijter H.M., Nelissen B.G.L., Bots M.L., Kappelle L.J., Rinkel G.J.E., et al., "Management of Extracranial Carotid Artery Aneurysm," Eur J Vasc Endovasc Surg. 50(2) 141-147 (2015).

[4] Sfyroeras G.S., Dalainas I., Giannakopoulos T.G., Antonopoulos K., Kakisis J.D., Liapis C.D., "Flow-diverting stents for the treatment of arterial aneurysms," J Vasc Surg. 56(3), 839-346 (2012).

[5] Amuluru K., Al-Mufti F., Singh I.P., Prestigiacomo C., Gandhi C., "Flow Diverters for Treatment of Intracranial Aneurysms: Technical and Clinical Updates," World Neurosurg. 85, 15-19 (2016).

[6] Baptista-Sincos A.P.W., Simplício A.B., Sincos I.R., Leaderman A., Neto F.S., Moraes A., et al., "Flow-diverting Stent in the Treatment of Cervical Carotid Dissection and Pseudoaneurysm: Review of Literature and Case Report," Ann Vasc Surg. 46, 372-379 (2018).

[7] Robijn S.M.M., Welleweerd J.C., Lo R.T.H., Moll F.L., de Borst G.J., "Treatment of an extracranial internal carotid artery aneurysm with a flow-diverting stent," J Vasc Surg Cases 1(2), 191-193 (2015).

[8] Viti J., Vos H.J., de Jong N., Guidi F., Tortoli P., "Detection of Contrast Agents: Plane Wave Versus Focused Transmission," IEEE Trans Ultrason Ferroelectr Freq Control 63(2), 203-210 (2016).

[9] Voorneveld J., Muralidharan A., Hope T., Vos HJ., Kruizinga P., van der Steen A.F.W., et al., "High Frame Rate Ultrasound Particle Image Velocimetry for Estimating High Velocity Flow Patterns in the Left Ventricle," IEEE Trans Ultrason Ferroelectr Freq Control 65(12), 2222 -2232 (2017).

[10] Engelhard S., Voorneveld J., Vos H.J., Westenberg J.J.M., Gijsen F.J.H., Taimr P., et al., "High-Frame-Rate Contrast-enhanced US Particle Image Velocimetry in the Abdominal Aorta: First Human Results," Radiology 289(1), 119-125 (2018).

[11] Voorneveld J., Engelhard S., Vos H.J., Reijnen M.M.J., Gijsen F., Versluis M., et al., "High Frame Rate ContrastEnhanced Ultrasound for Velocimetry in the Human Abdominal Aorta," IEEE Trans Ultrason Ferroelectr Freq Control 65(12), 2245-2254 (2018).

[12]Hoving A.M., Voorneveld J., de Vries E.E., Groot Jebbink E., Mikhal J., Versluis M., et al. "In vitro high-framerate contrast-enhanced ultrasound particle image velocimetry in a carotid artery stent," Proc. SPIE 10580 (2018).

[13] Jing L., Liu J., Zhang Y., Paliwal N., Meng H., Wang S., et al., "Analysis of Multiple Intracranial Aneurysms with Different Outcomes in the Same Patient after Endovascular Treatment," World Neurosurg. 91, 399-408 (2016).

[14] Shih T.C., Lin Y.H., Ho Y.J., Hsiao H.D., Huang Y.H., Huang T.C., "Hemodynamic analysis of vascular stenting treatment outcome: Computational fluid dynamics method vs optical flow method," J Xray Sci Technol. 20(4), 469-481 (2012). 
[15] van Bogerijen G.H.W., Auricchio F., Conti M., Lefieux A., Reali A., Veneziani A., et al., "Aortic Hemodynamics After Thoracic Endovascular Aortic Repair, With Particular Attention to the Bird-Beak Configuration," J Endovasc Ther. 21(6), 791-802 (2014).

[16] Mikhal J., Slump C.H., Geurts B.J., Simulation of pulsatile flow in cerebral aneurysms: from medical images to flow and forces. Aneurysm, ISBN: 978-953-51-0730-9, Y. Murai (Ed.), InTech, 199-222 (2012).

[17] Mikhal J., Kroon D.J., Slump C.H., Geurts B.J., "Flow prediction in cerebral aneurysms based on geometry reconstruction from 3D rotational angiography," Int j numer method biomed eng 29(6-7), 777-805 (2013).

[18] Conti M., Long C., Marconi M., Berchiolli R., Bazilevs Y., Reali A., "Carotid artery hemodynamics before and after stenting: A patient specific CFD study," Comput Fluids 140, 1-13 (2015).

[19] Mikhal J., Ong G.M., de Jong G., Aquarius R., de Vries J., Boogaarts J., Geurts B.J., "Toward automated analysis of flow in stented aneurysms," Proceedings CMFF 18, (2018).

[20] Attigah N., Külkens S., Zausig N., Hansmann J., Ringleb P., Hakimi M., et al., "Surgical Therapy of Extracranial Carotid Artery Aneurysms: Long-Term Results over a 24-Year Period," Eur J Vasc Endovasc Surg. 37(2), 127 133 (2009).

[21] Mikhal J., Geurts B.J., "Development and application of a volume penalization immersed boundary method for computation of blood flow and shear stress in model aneurysms," J Math Biol, 67(6-7), 1847-1875 (2013). 